\title{
Traffic Video Surveillance in Different Weather Conditions
}

\author{
Igor Vujovića, Marjan Jurčevićb, Ivica Kuzmanića
}

Various parameters are studied in video-stream traffic surveillance. Weather conditions are one of the most important parameters, influencing the quality of traffic surveillance in any microlocation, such as the town of Metković in this study. This paper examines the influence of weather conditions on traffic surveillance based on the collection of visual data. The quality of the system was tested under the following weather conditions: sunny, windy, cloudy and rainy.

\section{KEY WORDS}

$\sim$ Vehicle detection

$\sim$ Movement recording

$\sim$ Road traffic surveillance

$\sim$ Weather conditions a. University of Split, Faculty of Maritime Studies, Split, Croatia

e-mail: ivujovic@pfst.hr

b. Zrinsko-Frankopanska 82, Metković, Croatia

e-mail: charly_mtk@hotmail.com

\section{INTRODUCTION}

Traffic surveillance is a fast growing area of research. There are many methods, which address different problems in traffic surveillance. Traffic surveillance can be performed by different sensors in different situations and types of traffic, from air traffic separation (Williams et al., 2008) to marine integrated surveillance (Moutray and Ponsford, 1997).

Cutting-edge studies of traffic surveillance deal with components of intelligent surveillance systems and high-level modules of computer vision. However, advances have also been made in low-level processing. An example of both courses of research is provided in (Cucchiara et. al., 2000), where rule-based reasoning on visual data is used. The proposed system consists of several modules, which can be grouped into low-level and high-level. At high level, symbolic reasoning is introduced. At low level, two groups of modules are used: for daylight and nightlight. During the day, motion detection must be corrected with luminance variation detection. At night, morphology and headlight pairing is performed. Low-level problems were addressed i.e. in (Bhandarkar and Luo, 2005), where background updating is addressed or in (Chang, 2006), where shadow removal algorithm is proposed. High level problems were addressed i.e. in (Liu and Payeur, 2003), where activity detection was considered or in (Kumar et al., 2005), where behavior interpretation was considered. A method for vehicle count in the presence of occlusions was proposed in (Pang et al., 2007).

In this paper, we present road traffic surveillance experiments performed in the Metković region known for having weather conditions specific only to that region, making it reasonable and mandatory to examine and investigate the impact of weather conditions on the accuracy of traffic surveillance systems.

The paper is organized as follows. In the second section, a review of some recent research in traffic surveillance taking 
weather conditions into account is presented. The third section describes the setups and the experiments performed in this work. The forth section gives conclusions and proposes further research.

\section{TRAFFIC SURVEILLANCE UNDER DIFFERENT WEATHER CONDITIONS}

There are references dealing not only with traffic surveillance, but also including considerations about weather conditions. In this section, references are grouped as:

- examples of usage from practice (government and private),

- $\quad$ highway (freeway) surveillance, and

- urban traffic surveillance.

\subsection{Government and private sector efforts}

The Norwegian Public Roads Administration (NPRA) manages the country's roads for everything from weather conditions to accidents (Norwegian Roads Agency Deploys Hardened Wireless Network, 2012). This is important for traffic safety and emergency interventions, as well as for traffic pattern research relevant for planning further road network development.

There are also corporations offering complete traffic surveillance solutions, including weather conditions, which are intelligent and cover motorways, tunnels and waterways (Intelligent solutions for motorways, tunnels and waterways, 2010).

The Arizona Department of Transportation spends about $\$ 50,000$ per location for the installation of Freeway Management System (FMS) detection, and another $\$ 1,000$ per annum for the maintenance of each site. They used a list of starting points to establish the common conditions that need to be considered, such as: morning peak, afternoon peak, off-peak, dry weather, wet weather, congested conditions with slow speeds, free-flow conditions, intense fog, blowing dust, full sunlight, full dark, light transitions, and snow.

The (State of the Art Evaluation of Traffic Detection and Monitoring Systems, 2007) reports that the quantity of errors increases in wet weather. For example, while detected errors in dry weather do not exceed $20 \%$, they may rise up to as many as $65 \%$ in case of wet weather. The importance of these studies can be illustrated by investment in experimental setups.

Governments have also shown concern for weather conditions in traffic monitoring, and ordered the relevant studies. For example, the US Federal Highway Administration obtained results from the Road weather management program (Goodwin, 2003). The report illustrates best practices with examples from US roads.

An example of European private corporation study may be found in (Versavel, 2006). The report states that traffic surveillance problems are due to the fact that there are no standard camera positions, no standard roads, no standard weather and no standard illumination. It is also stated that different applications require different cameras and different camera positions. It has to be pointed out that the main problem in video traffic surveillance is the validity of the obtained traffic data, inevitably leading to the conclusion that weather conditions having to do with the cleanliness of the camera lens are the main issue. The influence of fog depends on the camera's position and density of the fog. If the camera is mounted above the highway and visibility is about $20 \mathrm{~m}$, than it will have no impact on the results of surveillance. On the other hand, rain and snow can influence work by making camera dirty if left unprotected from such conditions. However, right positioning and protection will reduce the effect of snow and rain. Also, it goes without saying that the same goes for dirt. The camera should be mounted at a sufficiently high position.

\subsection{Surveillance of highways}

The recognition of importance of weather conditions is outlined with research in (Lagorio et al., 2008). An analysis of different weather conditions is presented and solution proposed. The solution is based on the developed system, which uses a statistical framework (mixture of Gaussians, MoGs) to identify specific meteorological events, such as snow, fog or heavy rain. Of course, the above mentioned examples are used for surveillance of state and interstate roads, not for urban traffic.

(Nookola, 2006) introduced methodology for analyzing the impact of weather on traffic. The author used correlation coefficient analysis to establish which weather parameter affects traffic and daily traffic volume variability under different weather conditions.

(Arth et al., 2006) proposed an algorithm that performs reasonably well under adverse weather conditions, such as heavy rain or snow. Errors, however, occur due to shadows or occlusions. Furthermore, the algorithm is sensitive to camera motion, such as jitter. A further drawback is that objects, which become stationary, tend to fade into the background model. They used the approximated median filter for background modeling and Viola-Jones detector (Viola et al., 2001).

(Roh et al., 2013) proved that change in weather conditions triggers variations in highway traffic. The goal of the research was to use truck traffic as a model to plan efficient winter road maintenance programs.

All the above studies indicate that the study of the influence of weather conditions on traffic has practical applications.

\subsection{Urban traffic surveillance}

Dealing with urban traffic opens a variety of issues. Traffic congestions, common in urban areas and their vicinity, are 
managed by the intelligent transport system (ITS). ITS integrates traffic radars, loop detectors and surveillance cameras.

Two main difficulties in the establishment of the virtual loop detector (VLD) are changing illumination and weather conditions. Proposed solution in (Zhidong et al., 2008) increases average accuracy by $95 \%$ under changing illumination in various weather conditions in daytime in comparison to relevant previous research. Texture features in describing the traffic density are used in (Wassantachat, 2009). Background modeling is performed by the SVM kernel, which self-adapts to various lighting environments.

Urban traffic is more challenging due to:

- high traffic density,

- position of cameras, i.e. lower angles resulting in high occlusion rates, and

- variety of road users.

Furthermore, evaluation under challenging weather conditions would be desirable, but is rarely performed (Buch et al., 2011). Different techniques for urban road user detection and classification are proposed, such as in (Buch et al., 2010), where motion silhouettes were extracted and compared to projected model silhouettes. The comparison resolved the issue of the classification of motion silhouettes. Performances were tested under three different weather conditions and results were $87 \%$ better than in competitive algorithms.

One of the challenging problems in urban traffic is the tracking of vehicles at crossroads. In (Liwei at al., 2011), a new method is proposed. The method is innovative in the application of two-stage view selection and dual-layer handling of occlusions. The paper proposed a Multi-Modal Particle Filter (MMPF) for tracking vehicles explicitly. A considerable number of experiments were performed under different weather conditions. The considered weather conditions were snowy, sunny and cloudy.

(Smids, 2006) used webcams (in our example, webcam was also used) for urban traffic monitoring applying the background subtraction technique. The proposed system was evaluated under different weather conditions. The author compared the deterministic and statistical approach in background subtraction and background modeling. The author reported that deterministic subtraction approach had some important limitations. For example, total foreground area was often not fully detected, objects which became background were erroneously detected as foreground. However, results based on statistical approach using per-pixel Gaussian mixture model (GMM) were reported as very promising. The deterministic approach has no limitations. Sunny, cloudy and rainy weather conditions were used in the research. Experimental work was performed with a capture resolution of $640 \times 480$ and frame rate was $15 \mathrm{fps}$. The author is of the opinion that background should be adaptive. In non-recursive modeling techniques, a sliding window approach is used for that purpose. It depends on buffer length, n, and is given as:

$B_{t}(x, y)=F\left(I_{t-1}(x, y), \ldots, I_{t-n}(x, y)\right)$

where $B_{t}$ is the background at time $t, l_{t}(x, y)$ is the frame coming from the camera at time $t$ and $F$ is a function that is based on the temporal variation of pixel values. Function $F$ is obtained in a variety of ways: such as frame differencing, median filter, linear predictive filter or some non-parametric model. The simplest model is frame differencing, which leads to background definition as:

$B_{t}(x, y)=I_{t-1}(x, y)$

This, the simplest method, is only based on the previous frame. The calculation of the median for several frames in the buffer is a bit more complex:

$B_{t}(x, y)=\operatorname{median}\left(I_{t-1}(x, y), \ldots, I_{t-n}(x, y)\right)$

In linear predictive filters, background model is defined as:

$B_{t}(x, y)=\sum_{i=1}^{n} a_{i} I_{t-i}(x, y)_{i}$

where $a_{i}$ is the predictive coefficient.

The most popular recursive techniques mentioned are the approximated median, Kalman and mixture of the Gaussians.

(Smids, 2006) used Open Source Computer Vision Library (OpenCV) for the implementation of the proposed work. Finally, (Smids, 2006) concluded that regardless of the weather conditions, the statistical approach outperforms the deterministic approach in video surveillance applications. However, he reported a number of small misclassification intervals due to rainy weather.

(Zhou et al., 2007) reported, according to their experimental results based on real traffic video data, that their vehicle detector was excellent at dealing with different weather and illumination conditions. They based their conclusion on counted vehicles passing by the area under different weather and illumination conditions: afternoon sunlight with long and heavy shadow, morning sunlight with long and light shadow, a rainy day with reflections on the side of vehicles, nightfall with bright vehicle headlights but no streetlight and at night under streetlight with shadows and headlights. Their approach was the implementation of SVM (Support Vector Machine) in training. They obtained 
SVM-classifiers, which they used in further experimental work. The scatter matrix for PCA (Principal Component Analysis) is computed using a training sequence. Finally, they concluded that the experimental results derived from real traffic video data showed that their vehicle detector has an ability to deal with different weather conditions.

As stated in (Nandhini and Parthiban, 2012), a video-based monitoring system must be resistant to different weather and illumination conditions. The algorithm deals with shadows as well. The proposed algorithm includes lighting object extraction, connected-component extraction, spatial classification process, potential component tracking, motion-based grouping, tracking of vehicle component groups and vehicle identification and classification. In the research, satellite images were used. The preprocessing of such images consists of several operations, such as atmospheric correction, change detection, image registration or geometric correction. Vehicle lights were used as the silent feature for nighttime vehicle detection. Then morphology was used, as well as shape analysis, etc. Edge-based detection experiments were performed under different weather and lighting conditions. Finally, the authors concluded that their proposed algorithm can be used in real-world and real-time road traffic applications.

(Cheung and Kamath, 2005) are more concerned with the operation of the standard modules, which every reliable video surveillance system of differencing type must have - robust background subtraction. They validated their proposal for background subtraction in urban traffic video.

(Hu et al., 2008) focused on data fusion provided by different viewpoints and the probability of vehicle presence. They submitted that noise caused by weather, sudden light changes and weak shadows could be efficiently eliminated using their method. The authors used a concept of probability fusion map (PFM), which is the probability of a vehicle being present in the scene. PFM is calculated by merging mapped images from different cameras. The additional benefit of using more than one camera is the ability to obtain 3D information, such as length, width and height in the process of blob analysis. They proposed a key factor called PFM factor, which synthesizes the inverse projected images with considerations of Inverse Projector Factors (IPF) and Perspective Accuracy Factor (PAF). The IPF depends on the properties of the pixel and PAF is expressed by factor $\beta$ :

$\beta_{i}(x, y)=1 \kappa_{i} \log _{10} \frac{P_{i}(x, y)}{P_{i 0}}$

where $P_{i 0}$ is camera distance to the nearest feature point, $\mathrm{k}_{i}$ an adjustment weight parameter and $P_{i}(x, y)$ is the distance from target to camera calculated on the inverse projected map.
(Narasimhan and Nayar, 2003) concluded that there is no point in improving classic noise-removal techniques to remove the influence of weather from the image. The reason is in contrast decay, which is caused by weather. They developed a physicsbased model which describes the appearances of scenes in uniform bad weather conditions.

As can be seen, some papers concentrate on low level and some on high level processing of the input video stream. However, both low and high level processing have the potential for further research due to the above mentioned issues, which still remain unresolved/unresearched or not resolved in a satisfactory manner.

\section{EXPERIMENTAL SETUP AND RESULTS}

In this section, we described the performed experimental setup, results, and measures of quality. As previously stated, the Metković region is known for having weather conditions specific only to that region. So it is reasonable and mandatory to research and investigate the impact of weather conditions on the accuracy of the traffic surveillance systems. The quality of the system is examined for the following weather conditions: sunny, windy and cloudy, and rainy, since these weather conditions are the most frequent in the geographical microregion of the town of Metković, Croatia.

\subsection{Description of experimental setup}

Equipment used in the experiments is as follows: network camera AXIS 207, software surveillance application, laptop HP Compaq 6715b. Characteristics of the camera Axis 207 are as follows: dimensions $85 \times 55 \times 34 \mathrm{~mm}$, weight $177 \mathrm{~g}$, network connection RJ45, grid adapter $4 \mathrm{~W}$ power, and the input/ output terminal connector (Axis 207/207W/207MW Installation Guide, 2012). MJPG format was used for processing. Image resolution was $640 \times 480$ pixels. Network cameras make possible the organization of traffic surveillance in control centers. Such cameras are organized as free-standing and can be contacted by different authorized computers. The advantage is the possibility of networking many cameras into a single system.

Further advantages of the cameras used are (Axis net cameras, 2012):

- $\quad$ remote and safe access to live video stream and recorded videos,

- flexible and economic solutions to video control,

- more reliable identification,

- PAL/NTSC may be used, but even higher resolutions can be achieved,

- progressive scanning to remove blur,

- powerful event management with intelligent video features, 
- automatic reaction to events and threats, drastically reducing the number of operative personnel and raising the efficiency of video surveillance,

- scalable, easily integrated solution,

- open access technology platform,

- $\quad$ easy integration into other systems as access control or point of sale.

- lower total costs,

- power over ethernet is possible, reducing the costs of installation at remote locations, and

- connection to standard IP network/ethernet.

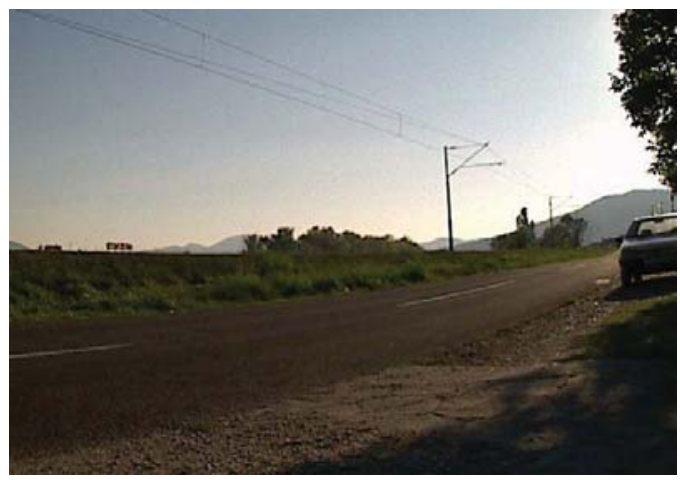

a)

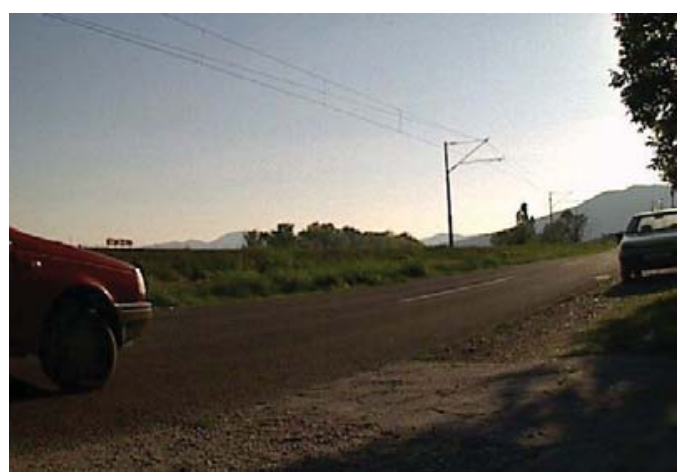

b)

\subsection{Results}

Camera was positioned at state road D62 in the vicinity of the town of Metković in the Republic of Croatia.

The first experiment was performed under sunny weather conditions. As shown in Section 2, weather conditions influence the performance of the systems. Therefore, different weather conditions have been experimentally studied. The examples of the results are shown in Figure 1.

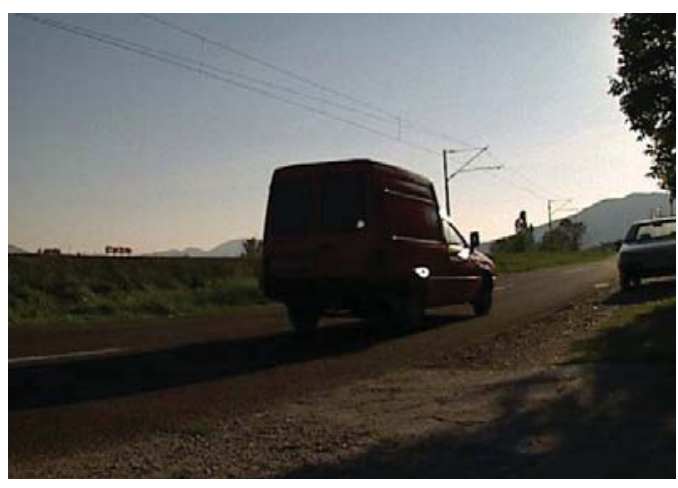

c)

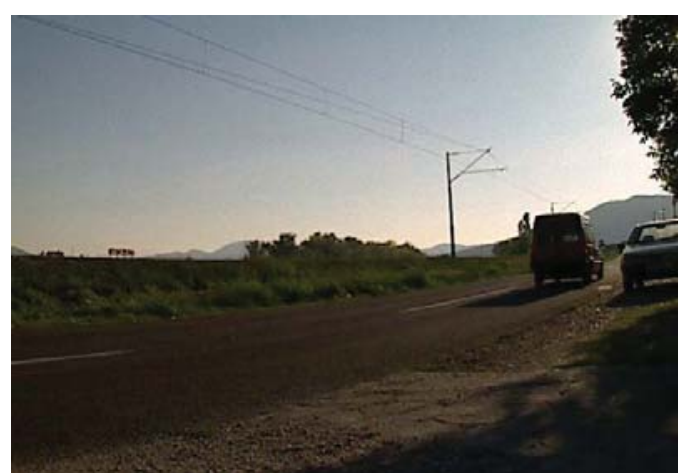

d)

Figure 1.

Motion detection in experiment 1 (camera is not directly exposed to the sun): a) no motion, b) c) d) motion detected.

In the experiment, the camera was tested in two modes of operation: the shooting of moving images (when there is no motion, no picture is not taken) and constant recording of traffic. Constant recording is useful if motion detector fails to trigger the recording device. It could be examined how well the motion detector and hardware operate in real conditions. Both software and hardware support both modes of operation.
The second experiment was performed in windy and cloudy weather. Generally known problems occurring under these weather conditions are: waving trees, thresholding, detection of cloud movement and so on. In some cases, some of the problems can be avoided by adding specific program routines. This solution cannot be used generally. 


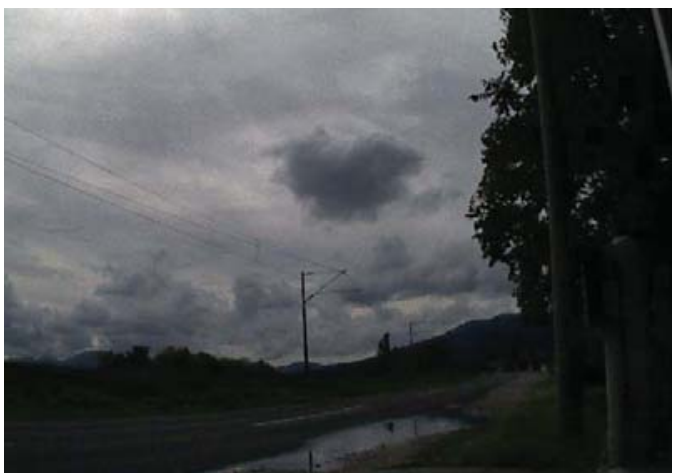

a)

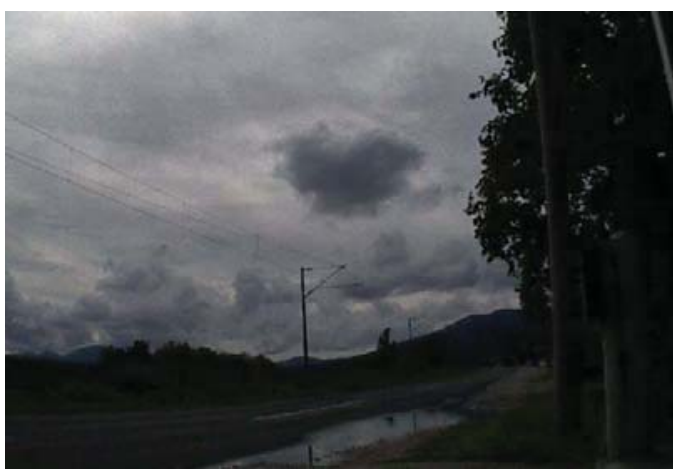

b)

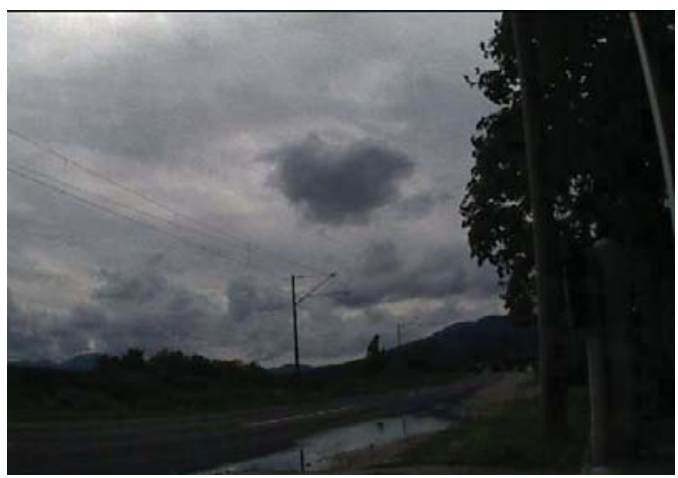

c)

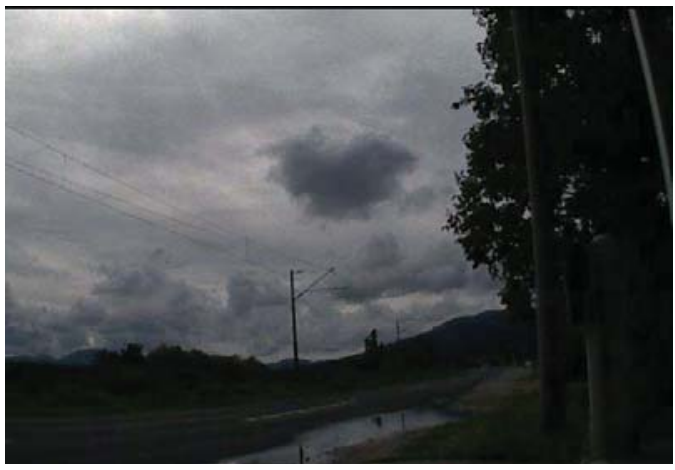

d)

Figure 2.

Motion detection under windy and cloudily weather: a) no motions, b), c), d) wrong detection of motions: waving trees effect.

The third experiment was performed in rainy conditions. In this case, detection of rain as motion presents a problem, which could be avoided by setting a proper threshold. Unfortunately,

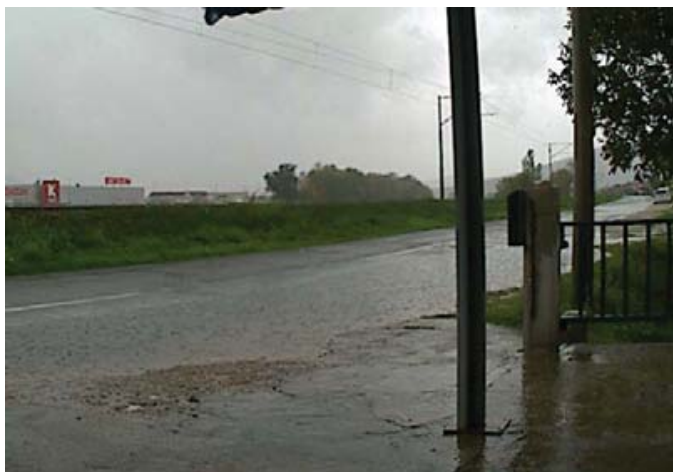

a) the camera does not have features which would allow it to differentiate between heavy rain and movement. Therefore, there is no rain sensibility.

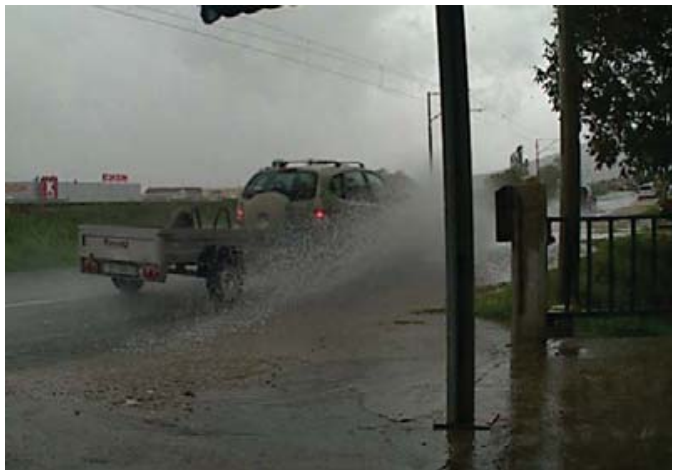

c) 


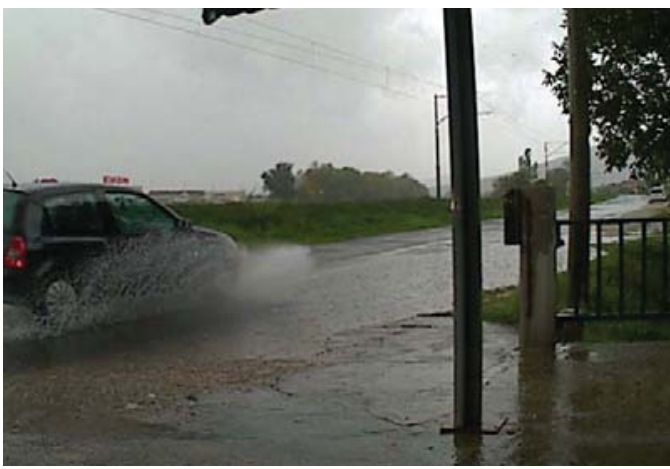

b)

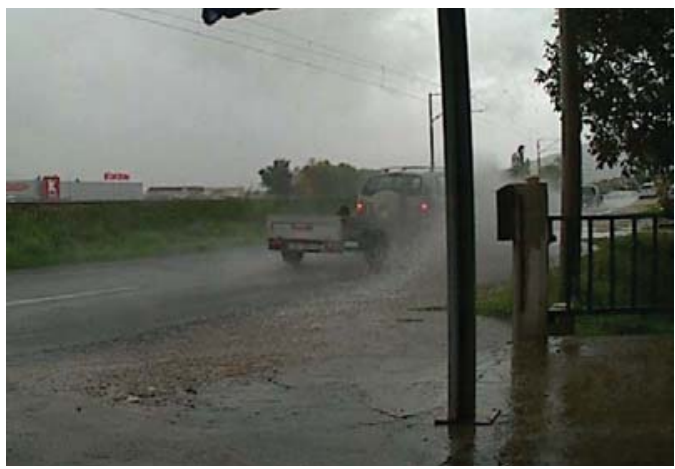

d)

Figure 3.

Motion detection during rain: a) rain as detected motion, b), c), d) real motion detection.

In order for there to be a noticeable difference, frame differencing has to be performed. The used application software enables frame differencing. The result is in Figure 4. Figures 4.a and 4.b show two neighboring frames in the video stream. Figure 4.c presents the difference between Figures 4.a and 4.b. It can be seen that frame differencing can produce double-vision effect in the motion mask. The result is presented in black and white technique. This type of image is called a motion mask. It is clear from the motion mask that shadow detection can be a very real problem when we want to use the obtained motion mask further in high level applications. The second problem of the application software used is the avoidance of double detection. A higher vision application, like car counting, can instigate false detection by mistake.

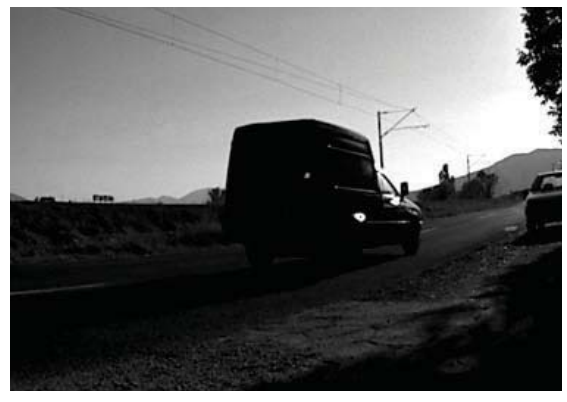

a)

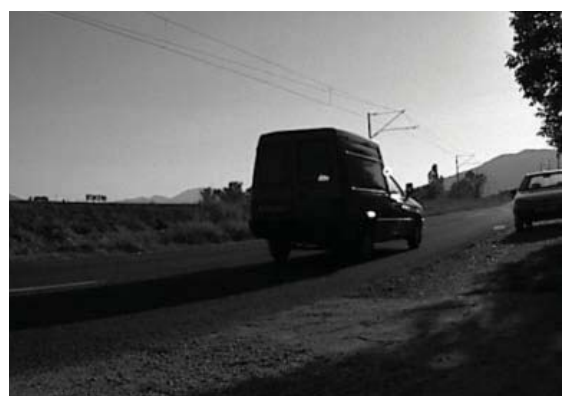

b)

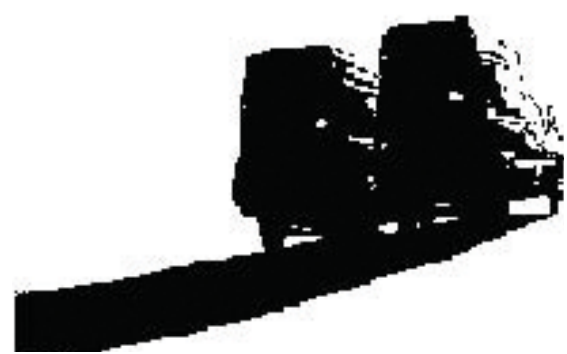

c)

Figure 4.

Frame differencing: a) frame 1, b) frame 2, c) motion mask obtained by frame differencing.

The result of image sequence processing is the image difference. Further research should include higher vision applications, i.e. traffic counting, which will enable traffic development planning. 


\subsection{Measures of quality}

Measures of quality can be divided into 11 groups based on:

- pixels,

- objects,

- spatial precision,

- temporal and spatio-temporal precision,

- estimation methodology,

- receiver-operating characteristics,

- perturbation detection rate,

- standalone performance evaluation,

- intra-object homogeneity metrics,

- inter-object disparity metrics, and

- combination of the above mentioned.

In pixel based quality measures, pixels are classified into four groups:

- true positives (TP), which means that the algorithm correctly detected and classified the pixel as foreground,

- false positives (FP), which means that the algorithm mistakenly classified a pixel as foreground instead of as background,

- true negatives (TN), which means that the algorithm correctly classified a pixel as background,

- false negatives (FN), which means that the algorithm classified a pixel as background instead of as foreground.

Based on the above mentioned definitions, several measures are introduced. The best known is the percentage of correct classifications (PCC) (Rosin and loannidis, 2003; Elhabian et al., 2008):

$P C C=\frac{T P+T N}{T P+T N+F P+F N}$

The second measure based on pixels is the so called Jaccard coefficient (JC), defined as:

$J C=\frac{T P}{T P+F P+F N}$

The third statistical measure is Yule's coefficient (YC):

$Y C=\left|\frac{T P}{T P+F P}+\frac{T N}{T N+F N}-1\right|$
False detection rate can be defined as:

$F D R=\frac{F P}{T P+F P}$

In some cases, an interesting measure can be false negative detection rate defined as:

$F N D R=\frac{F N}{T P+T N+F N+F P}$

The total number of false detections, total false detection rate, TFDR, is defined as:

$T F D R=\frac{F N+F P}{T P+T N+F P+F N}$

The issue of statistical pixel measurement is possibly misleading. It is especially noticeable in scenes containing changes small relative to the total number of pixels.

Although misleading in many situations, the pixel criterion is easy to implement, does not require high vision algorithms and high execution times.

For example, if we take Figure 4, we can measure that PCC is 0.74 , which is lower than many methods in video surveillance. However, since all of these methods should be evaluated under the same conditions, using the same scenes, this is nothing more than an assumption, not a scientific fact.

Further numerical result is the FNDR, which is only 0.021 , because we did not take shadows and double-detections into consideration. However, when shadows and double detections are taken into account, the appropriate measure, FDR, rises to 0.2381 or $23.8 \%$, which is a very bad result in comparison to the world's best results. TFDR is almost $26 \%$. One of the problems with real scenes and the evaluation of new experiments is the lack of grounds for comparison. There are not many publicly available and compatible source codes to compare with your scene. On the other hand, if the available reference video sequences are used, one risks being misled, because there is no guarantee that the obtained results can be applied to all scene in general.

Unfortunately, the research results from available literature focus on higher vision applications, such as traffic patterns, time predictions, speed predictions, etc., i.e. in (Roh et al., 2013). For example, vehicle counting is influenced by weather conditions, i.e. in (Zhou et al., 2007) rainy conditions contribute to false car counting by less than $1 \%$. Error rates increase by minimum $20 \%$ in humid weather (see State of the Art Evaluation of Traffic Detection and Monitoring Systems, 2007). 


\section{CONCLUSION}

The study of the influence of weather conditions was justified due to the specific climate characteristics of the Metković region. The goal of this study was to improve automatic traffic monitoring and control systems. The following weather condition were studied: sunny, rainy, and cloudy weather. The obtained results lead to the conclusion that the choice of the selection of the measure for the decision making process is fuzzy. A smart and intelligent system, containing event bases, must be included into research and development.

Experiments performed in the study revealed possible issues when dealing with outdoor applications of video surveillance, such as wrong detection of clouds as road movement, rain as motion or the waving tree effect.

The applications of road traffic surveillance are the study of drivers' behavior, traffic regulation violations, accident detection, traffic regulation, security, safety, and similar. Any of these accidents can be reported to the police or a monitoring station, and further actions can be taken under advisement. In the research, motion mask was the basis for the calculation of the measures of quality.

The experiments were performed as a part of requirements for a master's thesis (MSc). Further work could lead to the framework for the assessment of the influence of weather on traffic monitoring based on low-level image processing criteria. One of the future papers could deal with the calculation of the velocity of a moving object based on frame differencing. Possible issues could include slight camera vibrations or variations in position, which would result in an error in calculation. Small displacements can be caused by natural factors, i.e. the wind.

\section{REFERENCES}

Arth, C., Bischof, H., Leistner, C., (2006), TRICam - An Embedded Platform for Remote Traffic Surveillance, Proc. 2006 Conference on Computer Vision and Pattern Recognition Workshop (CVPRW'06), New York, June 17 - 22, pp. 125. http://dx.doi.org/10.1109/CVPRW.2006.208

Axis 207/207W/207MW Installation Guide, available at: http://www.axis.com/files/ manuals/27192.pdf, [accessed 5 October 2012.].

Axis net cameras, available at: http://www.axis.com/files/brochure/bc netcams_40664_hr_1009_lo.pdf, [accessed 2 October 2012.].

Bhandarkar S. M. and Luo, X., (2005), Fast and Robust Background Updating for Realtime Traffic Surveillance and Monitoring, Proceedings of the 2005 IEEE Computer Society Conference on Computer Vision and Pattern Recognition CVPR'05, San Diego, USA, June 25-25, IEEE Computer Society, pp. 55 - 55.

Buch, N., Orwell, J., and Velastin, S. A., (2010), Urban Road User Detection and Classification Using 3D Wire Frame Models, IET Computer Vision, 4(2), pp. 105 - 116. http://dx.doi.org/10.1049/iet-cvi.2008.0089

Buch, N., Velastin, S. A. and Orwell, J., (2011), A Review of Computer Vision Techniques for the Analysis of Urban Traffic, IEEE Transactions on Intelligent Transportation Systems, 12(3), pp. 920 - 939.,

\section{http://dx.doi.org/10.1109/TITS.2011.2119372}

Chang, F. L., (2006), Object Tracking, Shadow Removal and Collision Event Detection for Traffic Surveillance System, MSc Thesis, National Cheng Kung University, Taiwan.

Cheung, S. C. S. and Kamath, C., (2005), Robust Background Subtraction with Foreground Validation for Urban Traffic Video, EURASIP Journal on Applied Signal Processing, 14, pp. 2330-2340., http://dx.doi.org/10.1155/ASP.2005.2330

Cucchiara, R., Piccardi, M. and Mello, P., (2000), Image Analysis and Rule-Based Reasoning for a Traffic Monitoring System, IEEE Transactions on Intelligent Transportation Systems, 1(2), pp. 119-130., http://dx.doi.org/10.1109/6979.880969

Elhabian, S. Y., El-Sayed, K. M. and Ahmed, S. H., (2008), Moving Object Detection in Spatial Domain using Background Removal Techniques - State-of-Art, Recent Patents on Computer Science, 1(1), pp. 32-54. http://dx.doi.org/10.2174/10032

Goodwin, L. C., (2003), Best Practices for Road Weather Management, Report, Road Weather Management Program, Office of Transportation Operations, Federal Highway Administration.

Hu, Z., Wang, C. and Uchimura, K., (2008), 3D Vehicle Extraction and Tracking from Multiple Viewpoints for Traffic Monitoring by using Probability Fusion Map, Electronic Letters on Computer Vision and Image Analysis, 7(2), pp.110-119.

Intelligent Solutions for Motorways, Tunnels and Waterways, Siemens, (2010), available at: http://www.mobility.siemens.com/mobility/global/ SiteCollectionDocuments/en/road-solutions/interurban/intelligent-solutionsmotorways-tunnels-waterways-en.pdf, [accessed 14 November 2012.].

Kumar, P., Ranganath, S., Weimin, H. and Sengupa, K., (2005), Framework for Real-time Behavior Interpretation from Traffic Video, IEEE Transactions on Intelligent Transportation Systems, 6(1), pp. 43-53., http://dx.doi.org/10.1109/TITS.2004.838219

Lagorio, A., Grosso, E. and Tistarelli, M., (2008), Automatic Detection of Adverse Weather Conditions in Traffic Scenes, Proc. IEEE Fifth International Conference on Advanced Video and Signal Based Surveillance AVSS ‘08, Santa Fe, USA, September 1-3, Curran Associates, pp. $273-279$.

Li, Z., Tan, E., Chen, J. and Wassantachat, T., (2008), On Traffic Density Estimation with a Boosted SVM Classifier, Digital Image Computing: Techniques and Applications (DICTA), Canberra, Australia, December 1-3, IEEE Computer Society, pp. 117-123.

Liu, L., Xing, J. and Haizhou, A., (2011), Multi-view Vehicle Detection and Tracking in Crossroads, Proc. 2011 First Asian Conference on Pattern Recognition (ACPR), Beijing, China, November 28-28, pp. $608-612$.

Liu, Y. and Payeur, P., (2003), Vision-based Detection of Activity for Traffic Control, Proc. IEEE Canadian Conference on Electrical and Computing Engineering, Montréal,Canada, 2, May 4-7, pp. 1347 - 1350., http://dx.doi.org/10.1109/CCECE.2003.1226149

Moutray, R.E. and Ponsford, A.M., (1997), Integrated Maritime Surveillance (IMS) for the Grand Banks, MTS/IEEE Conference Proceedings OCEANS ‘97, October 6-9, 2, pp. $981-986$.

Nandhini, S. and Parthiban, P.G.S., (2012), Automatic Vehicle Detection during Nighttime Using Bright Pixel Segment with Spatial Temporal Technique, International Journal of Electronics and Computer Science Engineering, 1(3), pp. 1520-1525.

Narasimhan, S. G. and Nayar, S. K., (2003), Contrast Restoration of Weather Degraded Images, IEEE Transactions on Pattern 
Analysis and Machine Intelligence, 25(6), pp.713-724., http://dx.doi.org/10.1109/TPAMI.2003.1201821

Nookola, L. S., (2006), Weather Impact on Traffic Conditions and Travel Time Prediction, MSc Thesis, University of Minnesota Duluth.

Norwegian Roads Agency Deploys Hardened Wireless Network, Cisco Ltd., (2012), available at: http://www.cisco.com/en/US/prod/collateral/routers/ps380/ c36_703866_00_nra_cs.pdf, [accessed 14 November 2012.].

Open Source Computer Vision library, available at: http://www.intel.com/ technology/computing/opencv/, [accessed 14 November 2012.].

Pang, C.C.C., Lam, W.W.L. and Yung, N.H.C., (2007), A Method for Vehicle Count in the Presence of Multiple-Vehicle Occlusions in Traffic Images, IEEE Transactions on Intelligent Transportation Systems, 8(3),pp. 441-459., http://dx.doi.org/10.1109/TITS.2007.902647

Roh, H. J., Datla, S. and Sharma, S., (2013), Effect of Snow, Temperature and Their Interaction on Highway Truck Traffic, Journal of Transportation Technologies, 3, pp. 24-38., http://dx.doi.org/10.4236/jtts.2013.31003

Rosin, P. and Ioannidis, E., (2003), Evaluation of Global Image Thresholding for Change Detection, Pattern Recognition Letters, 24(14), pp. 2345-2356., http://dx.doi.org/10.1016/S0167-8655(03)00060-6

Smids, M., (2006), Background Subtraction for Urban Traffic Monitoring using Webcams, MSc Thesis, Universiteit van Amsterdam.

State of the Art Evaluation of Traffic Detection and Monitoring Systems, (2007), test report Texas Transportation Institute for Arizona Department of Transportation, available at: http://www.azdot.gov/tpd/atrc/publications/project_reports/pdf/ az627(1).pdf, [accessed 15 September 2012.].
UTP cable for LAN, available at: http://www.ic.ims.hr/faq/mreza/utp/utp.html [accessed 6 October 2012.].

Zhou, J., Guo, D. and Zhang, D., (2007), Moving Vehicle Detection for Automatic Traffic Monitoring, IEEE Transactions on Vehicular Technology, 56(1), pp. 51-59., http://dx.doi.org/10.1109/TVT.2006.883735

Versavel, J., (2006), Traffic Data Collection: Quality Aspects of Video Detection, Report, Traficon n.v., Bissegem, Belgium, available at: http://sites.poli.usp.br/ptr/ lemt/documents/07-0901.pdf, [accessed 10 October 2013.].

Viola, P. A. and Jones, M. J., (2001), Rapid Object Detection using a Boosted Cascade of Simple Features. In International Conference on Computer Vision and Pattern Recognition, Kauai, Hawaii, December 11-13, pp. 511-518.

Wassantachat, T., Li, Z., Chen, J., Wang, Y. and Tan, E., (2009), Traffic Density Estimation with On-line SVM Classifier, Proc. Sixth IEEE International Conference on Advanced Video and Signal Based Surveillance AVSS '09, Genova, Italy, September 2-4, Conference publishing Service, pp. 13-18.

Williams, J. T., McGraw, J. W., Pate, D. P. and Porter, S., (2008), Reducing Separation Requirements through Improved Navigation and Surveillance, Proc. Tyrrhenian International Workshop on Digital Communications - Enhanced Surveillance of Aircraft and Vehicles TIWDC/ESAV 2008, Island of Capri, Italy, September 3-5, pp. $1-6$. 